impression applicability and expressiveness. Remission is defined as target in therapy of SLE. According to SLEDAI and current treatment recommendations patients were divided in four groups of disease activity (Remission, Remission on treatment, LLDAS, active disease).

Results It was possible to include 72 SLE patients with 305 visits in the study. $64(88 \%)$ of them were female and 8 patients were male. Average age was $48(\mathrm{SD} \pm 13.1)$ and the media duration of lupus disease was 15.6 years (SD \pm 8.7). There was a significant correlation between MLS and ECLAM $(p<0.001)$, WAI $(p=0.027)$ and BDI $(p=0.003)$, whereas the SLEDAI and cSLEDAI just show correlation with BDI $(p=0.008)$, respectively $(p=0.042)$. Additionally, a correlation between MLS and the remission status was found $(\mathrm{p}<0.001)$.

Conclusions The MLS is a 10 minute easy to administer questionnaire in clinical routine. To be highlighted is that the MLS considers subjective health parameters, like Qol which might be relevant for disease treatment. Further results will be presented at the conference.

\section{P173 IS USE OF HYDROXYCHLOROQUINE ASSOCIATED WITH BETTER PATIENT REPORTED OUTCOMES IN LUPUS?}

Meenakshi Jolly, Vinay Sehgal. Rheumatology, Rush University, Chicago, USA

\subsection{6/lupus-2020-eurolupus.215}

Background Use of Hydroxychloroquine (HCQ) is known to be associated with less disease activity, flares, damage and better survival in patients with Systemic Lupus Erythematosus (SLE). It is not known if its use is also associated with better patient reported health outcomes, a core outcome in SLE.

Methods International data from Study on Outcomes of Lupus (SOUL) on LupusPRO, a patient reported disease targeted Quality of life (QOL) tool, from 2,157 patients with SLE, were compared based on use HCQ. Disease activity and damage were assessed using SELENA-SLEDAI and SLICC-ACR/ SDI. T tests and Chi square tests were used for comparisons. Linear regression analyses were undertaken with summary LupusPRO health (HRQOL), non-health (NHRQOL) and Lupus Impact Tracker (LIT) scores as the dependent variables, and HCQ as the independent variable. LIT scores were derived from LupusPRO data. Similar analysis for damage was also conducted.

Results Mean age was $40.5 \pm 12.8$ years. Ninety-three percent were women. HCQ use 798/2157 (37\%) was inversely associated with age, Asian race, disease duration, lupus nephritis, neurological and hematologic manifestations, and damage. HCQ use was directly associated with presence of photosensitivity. On univariate analysis, HCQ use was associated with better QOL ( $\beta$ 6.19, 95\% CI 4.15, 8.24, P $\leq 0.001$ for LupusPRO-HRQOL) and less impact on daily life ( $\beta-9.37,95 \%$
CI $-12.24,-6.50, \mathrm{P} \leq 0.001$ for LIT). Other predictors for QOL and LIT were age, education, Asian race and disease activity. On multivariate analyses (adjusted for age, education, Asian status) use of HCQ was independently associated with better outcomes (LupusPRO-HRQOL and LIT). However, addition of disease activity variable to the models resulted in loss of independent association of HCQ use with better outcomes, suggesting mediation through disease activity (table 1). Similarly, mediation was seen for beneficial effects of HCQ use on damage through disease activity.

Conclusions HCQ use in SLE is associated with better health outcomes (LupusPRO-HRQOL, impact on daily life, damage), and the effects are mediated through disease activity modification.

Acknowledgements Behalf of SOUL group.

\section{P174 EXTENDED ARTERIAL ULTRASOUND REVEALING INCREASED INTIMA MEDIA THICKNESS AND RELATION TO IMPAIRED MICROCIRCULATION IN SYSTEMIC LUPUS ERYTHEMATOSUS}

1,2 Christina Svensson, ${ }^{3}$ Hanna Jonasson, ${ }^{3}$ Tomas Strömberg, ${ }^{4} \mathrm{Per}$ Eriksson, ${ }^{4}$ Christopher Sjöwall, ${ }^{1,2}$ Helene Zachrisson. 'Dept. of Clinical Physiology, Linköping University Hospital, Linköping; ${ }^{2}$ Dept. of Medical and Health Sciences, Linköping University, Linköping; ${ }^{3}$ Dept. of Biomedical Engineering, Linköping University, Linköping; ${ }^{4}$ Rheumatology/Division of Neuro and Inflammation Sciences, Dept. of Clinical and Experimental Medicine, Linköping University, Linköping, Sweden

\subsection{6/lupus-2020-eurolupus.216}

Background Systemic lupus erythematosus (SLE) is a chronic inflammatory disease characterized by multiple organ involvement. Atherosclerosis is the underlying cause of SLE-related cardiovascular disease. With high frequency ultrasound it is possible to differ between atherosclerosis and inflammatory findings in the vessel wall. Our hypothesis is that both macroand microcirculation are impaired in SLE.

Methods Sixty patients (52 women, 8 men), range 23-63 years, classified with SLE according to the 2012 SLICC criteria, and 60 healthy controls (52 women, 8 men), range 23-63 years, were investigated. Intima-media thickness (IMT) was recorded with high frequency ultrasound (GE Logic E9) in common carotid arteries (CCA), common femoral arteries (CFA) and the aortic arch. Microcirculatory oxygen saturation was assessed with EPOS (Enhanced Perfusion and Oxygen Saturation) (PeriFlux 6000, Perimed, Järfälla, Sweden). The EPOS system measures red blood cell tissue fraction, speed resolved perfusion and oxygen saturation in the microcirculation of the skin.

Results IMT in common carotid artery (CCA) was $0.56 \pm 0.10$ $\mathrm{mm}$ in SLE patients vs $0.54 \pm 0.13 \mathrm{~mm}$ in healthy controls (ns). IMT in common femoral artery (CFA) was $0.58 \pm 0.24$ $\mathrm{mm}$ in SLE patients vs $0.48 \pm 0.12 \mathrm{~mm}$ in healthy controls

Abstract P173 Table 1 Mediation of effects of HCQ use with outcomes through disease activity

\begin{tabular}{|c|c|c|c|c|c|c|c|c|c|c|c|c|}
\hline \multicolumn{7}{|c|}{ LIT } & \multicolumn{6}{|c|}{ LupusPRO-HRQOL } \\
\hline & \multicolumn{3}{|c|}{ Univariate } & \multicolumn{3}{|c|}{ Multivariate } & \multicolumn{3}{|c|}{ Univariate } & \multicolumn{3}{|c|}{ Multivariate } \\
\hline Variable & B & $95 \% \mathrm{Cl}$ & p-value & B & $95 \% \mathrm{Cl}$ & p-value & B & $95 \% \mathrm{Cl}$ & p-value & B & $95 \% \mathrm{Cl}$ & p-value \\
\hline Activity (SLEDAI) & 2.040 & $0.90,3.18$ & $\leq 0.001$ & 2.830 & $1.25,4.40$ & $\leq 0.001$ & -1.84 & $-2.70,-0.97$ & $\leq 0.001$ & -2.29 & $-3.59,-0.98$ & 0.001 \\
\hline $\mathrm{HCQ}$ & -9.370 & $-12.24,-6.50$ & $\leq 0.001$ & -3.050 & $-7.93,1.84$ & 0.221 & 6.190 & $4.15,8.24$ & $\leq 0.001$ & 3.640 & $-0.46,7.73$ & 0.080 \\
\hline
\end{tabular}

